RESEARCH ARTICLE

\title{
Molecular Methods and Culture in Diagnosis of Tuberculous Meningitis in Children
}

\author{
Gaurav Sharma ${ }^{1,2}$, Bharti Malhotra', P. J. John², Shipra Bhargava ${ }^{3}$ \\ ${ }^{1}$ Department of Microbiology and Immunology, SMS Medical College, Jaipur, Rajasthan, India \\ ${ }^{2}$ Department of Zoology, University of Rajasthan, Jaipur, Rajasthan, India \\ 3 International Centre for Excellence in Laboratory Training (ICELT), National Tuberculosis Institute, Bengaluru, India
}

\begin{abstract}
Objectives: This study aimed to detect the incidence of tuberculous meningitis (TM) in pediatric patients using GenoType MTBDRplus, GenoType MTBDRsl, and Mycobacteria Growth Indicator Tube (MGIT). Additionally, it aimed to evaluate the utility of GeneXpert MTB/RIF assay in the detection of Mycobacterium tuberculosis in comparison to MGIT culture.
\end{abstract}

Methods: Children under or equal to 15 years of age who were clinically suspected of tuberculous meningitis (TBM) were included in the study. Cerebrospinal Fluid (CSF) samples were collected and tested for GeneXpert MTB/RIF assay and MGIT culture. Culture-positive samples were further analyzed using Line Probe Assay (LPA) to detect drug-resistant mycobacteria.

Results: Out of 272 CSF samples, 28 (10.3\%) samples were positive for MTB, and 5 (17.8\%) were MDRTB. GeneXpert MTB/RIF assay detected 23 (8.4\%) MTB cases of which 3 (13\%) were rifampicinresistant. MGIT culture detected the presence of MTB organism in 19 (6.9\%) cases, of which 6 (31.6\%) were isoniazid-resistant and 2 (10.5\%) were rifampicin-resistant by first line LPA. None of the samples had Extensively Drug-Resistant TB (XDR-TB). GeneXpert MTB/RIF assay had $73.6 \%$ sensitivity and 96.4\% specificity taking MGIT as a gold standard.

Conclusion: Total positivity for MTB was seen in $10.3 \%$ cases, among which $17.8 \%$ were MDR-TB; no XDR-TB was detected in pediatric patients. GeneXpert MTB/RIF assay is a rapid and reliable method for diagnosis of tuberculous meningitis but may miss some cases, so samples should also be cultured in MGIT to enhance yield and for extended sensitivity panel. J Microbiol Infect Dis 2021; 11(3):140-146.

Keywords: Tuberculous meningitis, pediatric, CSF, GeneXpert, MGIT

\section{INTRODUCTION}

Tuberculous meningitis (TBM) constitutes 1$5 \%$ of tuberculosis cases [1]. TBM patients may develop neurological disorders and other complications of multi-drug resistance in Mycobacterium tuberculosis which causes mortality in $20 \%$ to $22 \%$ cases [2]. So it is essential to diagnose TB and MDR-TB in pediatric patients at the earliest to give appropriate therapy timely [3]. Delay in diagnosis and treatment can lead to death [3]. Excellent and prompt diagnosis of tuberculous meningitis in children is very challenging, particularly in resource-limited settings due to paucibacillary nature, problems in collecting an adequate volume of CSF, and obtaining a detailed history [4]. Acid Fast Bacilli Staining (AFB) staining has poor sensitivity, and culture takes 2-6 weeks to provide results and has 35$60 \%$ sensitivity.

In 2013, WHO recommended using GeneXpert MTB/RIF assay for the diagnosis of TB in children and extra-pulmonary specimens [5]. GeneXpert MTB/RIF assay was strongly recommended by WHO in 2015 as an initial diagnostic test in paucibacillary disease in

Correspondence: Prof. Dr. Bharti Malhotra, Department of Microbiology and Immunology, SMS Medical College \& Hospital, JawaharLal Nehru Marg, Jaipur-302004, India E-mail: drbhartimalhotra@gmail.com 
preference to conventional microscopy \& culture [6]. It is a nucleic acid amplification testing that simultaneously detects $M$. tuberculosis and susceptibility to rifampicin and results in approximately two hours [7]. Studies have reported GeneXpert MTB/RIF assay sensitivity to vary from $58-80 \%[5,6]$.

The GenoType MTBDRplus assay (Hain Lifescience, Nehren, Germany) version 2 is a Line Probe Assay (LPA) based on a reverse hybridization procedure. It can be performed on smear-positive and smear-negative pulmonary samples and culture-positive isolates. LPA targets the rpoB, katG, and inhA genes, detecting $M$. tuberculosis and susceptibility to Rifampicin and Isoniazid drug, providing results in five hours [8].

This study aimed to detect the incidence of tuberculous meningitis and drug resistance in pediatric patients using GenoType MTBDRplus, GenoType MTBDRsI, MGIT, and evaluate the utility of GeneXpert MTB/RIF assay in the detection of Mycobacterium tuberculosis in comparison to MGIT culture.

\section{METHODS}

This study was conducted at Advance Research \& TB laboratory, Department of Microbiology, Sawai Man Singh Medical College, Jaipur. Specimens were collected from pediatric patients ( 15 years old or younger) clinically suspected of tuberculous meningitis from January 2018 to June 2018. Patient details were entered in Annexure 15A as per the Revised National Tuberculosis Control Program (RNTCP). This study was approved by the Ethics Committee S.M.S. Medical College \& Attached Hospitals, Jaipur (No. 2259/MC/EC/2016).

\section{Acid Fast Bacilli Staining and Microscopy}

AFB staining and microscopy were performed to detect mycobacteria as per the RNTCP guidelines [9]. One loopful of CSF was placed in the middle of a slide without spreading and allow to dry in the air. One more drop of CSF was placed on the same spot and allowed to dry. After centrifugation, a third drop of the deposit was placed on the same spot, airdried, and fixed. It was poured the filter carbol fuschin (1\%) to cover the smear. The slide was heated to keep the slide steamed for 5 minutes and washed off the stain with distilled water. Flooded slide with 3\% acid alcohol and was let stand for 2-3 minutes. Washed off the acid- alcohol with distilled watered and tilted the slide to drain. Flooded slide with methylene blue $(0.1 \%)$ and was let stand for 1 minute. The stain was washed off with distilled water, and the slide tilted to drain. The slide was allowed to air-dry. Examined the slide under a microscope using a $40 \mathrm{X}$ lens to select the suitable area and then examined under a 100X objective lens using a drop of immersion oil.

\section{GeneXpert MTB/RIF assay}

GeneXpert MTB/RIF assay was conducted as per the manufacturer's instructions [10]. One $\mathrm{ml}$ of the specimen was thoroughly mixed with $2 \mathrm{ml}$ of buffer solution; the mixture was then vortexed and incubated at room temperature for $10-15$ minutes. After that, $2 \mathrm{ml}$ of sample reagent mixture was transferred into GeneXpert MTB/RIF assay cartridge, scanned the cartridge, and loaded into the GeneXpert machine. Results were obtained after 2 hours. The systems reported the presence or absence of MTB with the bacterial load as very low, low, medium, or high and simultaneously giving results for susceptibility to rifampicin drug [11].

\section{MGIT Culture}

Five hundred microliter of CSF specimen was directly inoculated into MGIT (Mycobacteria Growth Indicator Tube) supplemented with eight hundred microliter OADC (Oleic acid, albumin, dextrose, catalase) containing PANTA (polymyxin B, amphotericin B, nalidixic acid, trimethoprim, azlocillin). Then it was placed into BACTEC MGIT 960 instrument and incubated at $37^{\circ} \mathrm{C}$ [12]. Decontamination was done in the case of turbid CSF. Positive tubes flagged by the instrument were removed and checked for the presence of mycobacteria by AFB staining \& microscopy. Contamination was checked by inoculating one drop of culture on a $\mathrm{BHI}$ agar plate and incubated for 48 hours at $37^{\circ} \mathrm{C}$. The instrument flagged negatives tubes after 42 days of incubation. The MPT 64 antigen test was performed on AFB positive MGIT cultures as described by the manufacturer [13]. This rapid immunechromatography test differentiates Mycobacterium tuberculosis and Nontuberculous Mycobacteria (NTM) [14].

\section{DNA extraction}

AFB positive MGIT cultures were subjected to DNA extraction using the GenoLyse kit (Hain Lifescience, Nehren, Germany) as per the 
manufacturer's protocol [15]. They were transferred $1 \mathrm{ml}$ of growth into a labeled $1.5 \mathrm{ml}$ screw-cap tube from AFB positive MGIT culture tube. The samples were centrifuged for 15 minutes at $10,000 \times \mathrm{g}$ in a standard tabletop microcentrifuge with an aerosol tight rotor. After that, the supernatants were discarded and resuspend pellet in 100 microliter Lysis Buffer (A-LYS) by vortexing. They were incubated for 5 minutes at $95^{\circ} \mathrm{C}$ in a water bath and briefly were spin down. In the end, 100 microliter Neutralization buffers (ANB) were added and vortexed sample for 5 seconds.

\section{Line probe assay}

Reverse hybridization was performed by using GenoType MTBDRplus V2 kit (Hain Lifescience $\mathrm{GmbH}$, Nehren, Germany) for molecular detection of MTB complex and resistance-conferring mutations to rifampicin (RIF) and isoniazid (INH) [15]. A positive Mycobacterium tuberculosis control (TUB) band indicated members of the $M$. tuberculosis complex. Rifampicin resistant samples were further subjected to second-line LPA; the GenoType MTBDRsl V2 (Hain Lifescience $\mathrm{GmbH}$, Nehren, Germany), for the detection of Extensively Drug-resistant Tuberculosis (XDRTB), by detecting resistance-conferring mutations of fluoroquinolones (FLQ) ( $g y r A$ and gyrB genes) and second-line injectable drugs (SLID) (rrs and eis genes) [16]. The absence of Wild type band \& presence of MUT band indicates the presence of mutation indicating resistance to the drug. Hetero-resistant strains showed both Wild \& MUT bands to the particular drug.

\section{Statistical analysis}

Statistical analysis was done to calculate the sensitivity, specificity, positive predictive value (PPV), and negative predictive value (NPV) of GeneXpert MTB/RIF assay versus MGIT culture considered a gold standard. Calculations were done with the help of an online calculator:

(https://www.medcalc.org/calc/diagnostictest.p hp).

\section{RESULTS}

Out of 272 CSF specimens tested, 23 (8.45\%) were found to be MTB positive, and 3 $(13.04 \%)$ were rifampicin-resistant by
GeneXpert MTB/RIF assay, 19 (6.98\%) samples were culture positive (Table 1).

On comparing the GeneXpert MTB/RIF assay and MGIT culture, 14 samples $(5.14 \%)$ were found positive by both, 9 (3.3\%) were GeneXpert MTB/RIF assay positive but culture-negative, 5 (1.83\%) were culture positive, but GeneXpert MTB/RIF assay negative. The comparative results were listed in Table 2.

On analyzing the results of first-line LPA, 4 $(21 \%)$ isolates were mono-resistant to isoniazid, and $2(10.5 \%)$ isolates were resistant to both rifampicin and isoniazid drugs. Among 23 MTB. GeneXpert positive cases, three were rifampicin-resistant, of which one was found to be MTB sensitive by LPA, and no mutation was observed in the RRDR region of the $r p o B$ gene. LPA showed the various mutation band patterns associated with the drug resistance in the $r p o B$, $k a t G$, and inhA gene shown in Table 3.

Among 19 culture-positive isolates, two isolates were found MDR by LPA. The $r p o B$ gene showed the mutation in the S531L codon, and a high level of isoniazid resistance was observed in the S315T1 codon of the katG gene. Another two isolates were monoresistant to isoniazid with high-level resistance in the S315T1 codon. A low isoniazid resistance was found in two isolates with the inhA gene C15T codon mutation.

The second line LPA was performed for two MDR-TB isolates and four INH resistant isolates obtained by first line LPA. Only one isolate out of two MDR-TB isolates showed the presence of mutation band pattern WT3MUT3C+ in the gyrA gene, which indicated the resistance to fluoroquinolone group (moxifloxacin, ofloxacin \& levofloxacin) while the remaining one and other four mono $\mathrm{INH}$ resistant isolates were sensitive to both fluoroquinolones and second-line injectable drugs (kanamycin, amikacin, capreomycin, and viomycin). In this study, no XDR-TB was detected, but pre-XDR-TB was detected.

\section{DISCUSSION}

Detecting MTB in patients suspected of tuberculous meningitis is a challenge due to the paucibacillary nature of tuberculous meningitis and the availability of minimal sample volumes of CSF. 
In this study, we observed total positivity of $10.3 \%$ in pediatric patients suspected of tuberculous meningitis. GeneXpert MTB/RIF assay showed $8.4 \%$ positivity comparable with the other multi-centric Indian study [17], which reported $7.1 \%$ positivity for CSF by GeneXpert MTB/RIF assay in a pediatric population. In this study, MGIT culture positivity was $6.9 \%$ lower than another study from Agra, India, by
Bhatia et al. [18], which showed $14.71 \%$ culture positivity. In this study, GeneXpert MTB/RIF assay detected MTB in $2.27 \%$ additional specimens then MGIT culture but missed $1.8 \%$ MTB which MGIT additionally detected. AFB smear microscopy did not detect acid-fast bacilli, similar to findings from another Indian study of Bala from Udaipur, Rajasthan [19].

Table 1. GeneXpert MTB/RIF assay results for CSF samples

\begin{tabular}{|c|c|c|c|c|}
\hline \multirow[t]{2}{*}{ Total Samples } & \multirow[t]{2}{*}{ MTB Positive (\%) } & \multicolumn{3}{|c|}{ MTB Detected } \\
\hline & & $\begin{array}{l}\text { Rifampicin } \\
\text { Sensitive (\%) }\end{array}$ & $\begin{array}{l}\text { Rifampicin Resistant } \\
(\%)\end{array}$ & $\begin{array}{c}\text { Rifampicin Indeterminate } \\
(\%)\end{array}$ \\
\hline 272 & $23(8.4)$ & $3(13 \%)$ & $20(87 \%)$ & $0(0 \%)$ \\
\hline
\end{tabular}

MTB=Mycobacterium tuberculosis

Table 2. Comparison of GeneXpert MTB/RIF assay by using MGIT culture as a gold standard

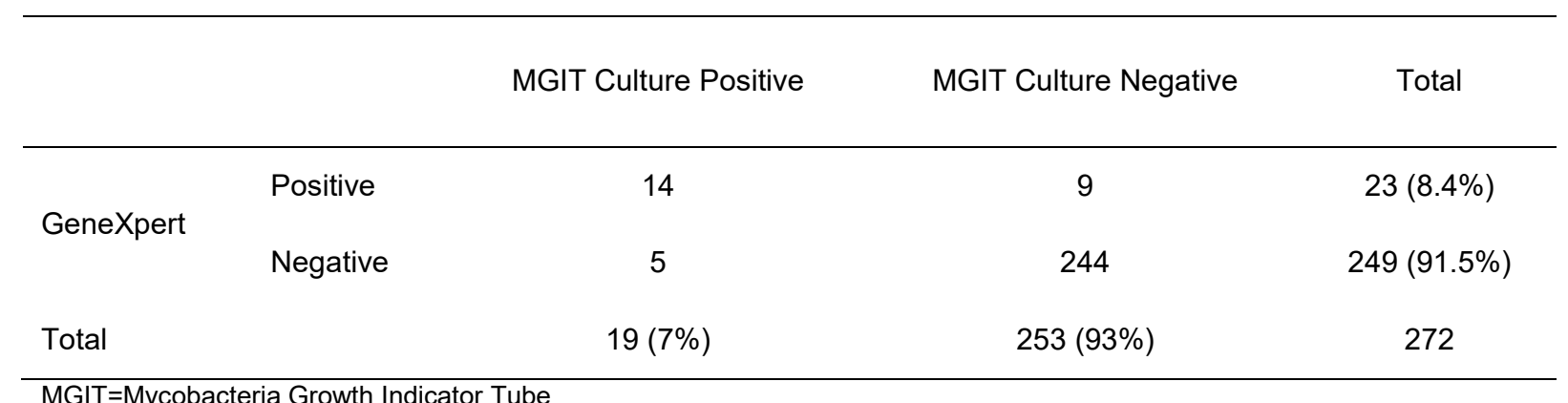

MGIT=Mycobacteria Growth Indicator Tube

Table 3. Frequency and pattern of rpoB, katG, and inhA gene mutations in drug-resistant Mycobacterium tuberculosis isolated from meningitis patients by LPA.

\begin{tabular}{|c|c|c|c|c|c|c|c|}
\hline \multicolumn{4}{|c|}{ Rifampicin } & \multicolumn{4}{|c|}{ Isoniazid } \\
\hline $\begin{array}{l}\Delta \mathrm{WT} \\
\text { probe }\end{array}$ & $\begin{array}{l}\text { rpo } B \text { gene } \\
\text { mutation }\end{array}$ & Codon & $\begin{array}{l}\text { No. of } \\
\text { isolates }\end{array}$ & $\begin{array}{l}\Delta \mathrm{WT} \\
\text { probe }\end{array}$ & $\begin{array}{l}\text { INH gene mutations } \\
\text { katG/inhA }\end{array}$ & Codon & $\begin{array}{l}\text { No. of } \\
\text { isolates }\end{array}$ \\
\hline \multirow[t]{2}{*}{ - } & MUT 3 & S531L & 2 & - & $\begin{array}{l}\text { MUT } 1 \\
\text { (katG) }\end{array}$ & S315T1 & 4 \\
\hline & & & & - & $\begin{array}{l}\text { MUT } 1 \\
\text { (inhA) }\end{array}$ & C15T & 2 \\
\hline
\end{tabular}

WT=Wild Type, INH=Isoniazid

Our findings showed that GeneXpert MTB/RIF assay had $73.6 \%$ sensitivity and $96.4 \%$ specificity for detecting TBM, using MGIT as the gold standard, similar to another study 
from Pondicherry, India [20]. In addition, they observed that GeneXpert MTB/RIF assay had $72.7 \%$ sensitivity and $98.5 \%$ specificity against culture.

Kohli et al. [21] from Canada reported the sensitivity and specificity of GeneXpert MTB/RIF assay against culture to be $71.1 \%$ and $98 \%$, respectively. Notably, of 23 cases detected by GeneXpert MTB/RIF assay, nine were not detected by MGIT culture. The socalled false positive (GeneXpert MTB/RIF assay positive but culture-negative) may occur due to loss of viability of MTB during decontamination of turbid CSF before culture [22]. Moreover, false negatives may also be due to loss of viability in patients under treatment. Out of 19 cases detected by MGIT culture, five isolates were not detected by GeneXpert MTB/RIF assay may be due to unequal splitting of the sample with low bacillary load $(<100 \mathrm{CFU} / \mathrm{ml})$ in CSF samples [23].

In this study, $17.8 \%$ MDR-TB was detected using both methods, $13 \%$ Rifampicin resistance was detected by GeneXpert MTB/RIF assay, which is comparable with another Indian study from Kolkata [24], which showed $13.8 \%$ resistance to rifampicin. In this study, GeneXpert MTB/RIF assay gave a better performance in detecting rifampicin resistance as it detected three RIF resistant while LPA detected two RIF resistant isolates and one isolate showed discrepant results, resistant by GeneXpert MTB/RIF assay but sensitive by LPA. As per RNTCP GeneXpert, MTB/RIF assay should be repeated in the discrepant sample, but we could not do so due to the unavailability of the sample. Two samples were negative by GeneXpert MTB/RIF assay. However, culture-positive were RIF sensitive with a low level of isoniazid resistance, as mutation was observed in the C15T codon of the inhA gene. So, each technology has its advantages; GeneXpert MTB/RIF assay detected more MTB and RIF resistant cases than MGIT and LPA but could not detect isoniazid resistance and missed few cases of MTB.

In the present study, LPA detected MDR-TB in $10.5 \%$ isolates which were comparable with another study from South Africa [25] which showed $11.6 \%$ MDR-TB in pediatric meningitis. $21.05 \%$ isolates were isoniazidresistant by first line LPA in our study, which agrees with another study from Spain [26] that reported $20.4 \%$ isoniazid resistance in pediatric patients. The paucibacillary nature of the disease in the pediatric population makes the diagnosis \& reporting of MDR/XDR difficult.

Only $1 / 2(50 \%)$ isolate was found resistant to the fluoroquinolones group. Thus, no XDR-TB was observed in our study, which is similar to another Indian study in which no XDR-TB was reported in pediatric patients with meningitis [27].

Though WHO-recommended GeneXpert MTB/RIF assay and LPA for detecting MDRTB, which identifies mutations in the $81 \mathrm{bp}$ RRDR region of the $r p o B$ gene, some of the mutations may be present outside this region and will be missed by both. Similarly, MGIT culture sensitivity which is the gold standard may also miss out on rifampicin resistance due to 'disputed' mutations [28]. The limitation of this study was the lack of adequate CSF volume in some specimens, as a result of which the required volume of a sample could not be put for MGIT culture after GeneXpert MTB/RIF assay testing. Treatment history was also not available in all cases.

\section{Conclusion}

Mycobacterium tuberculosis was detected in $10.3 \%$ of cases and MDR-TB in $17.8 \%$ of cases using all technologies. No XDR-TB was observed. GeneXpert MTB/RIF assay is highly effective in rapid and simultaneous detection of MTB and MDR-TB, which can help save lives and prevent complications by giving early treatment and management. Samples should also be cultured on MGIT to enhance the yield and carry out an extended panel of drug susceptibility as GeneXpert MTB/RIF assay can report only rifampicin resistance.

\section{ACKNOWLEDGMENTS}

All authors have extensively helped in writing and editing this manuscript. The final manuscript was seen and accepted by all authors.

Declaration of Conflicting Interests: The authors declare that they have no conflict of interest.

Funding: The authors are thankful to Foundation for Innovative and New Diagnostics (FIND) and National Tuberculosis 
Elimination Program (NTEP) for supporting work and providing financial assistance.

Authors' contributions: The manuscript was scrutinized for its intellectual content and the final version approved by all authors.

Mr. Gaurav Sharma carried out practical work and drafted the manuscript.

Dr. Bharti Malhotra conceived the work and corrected the manuscript.

Dr. P. J. John corrected the manuscript.

Dr. Shipra Bhargava carried out practical work and corrected the manuscript.

\section{REFERENCES}

1. Ducomble T, Tolksdorf K, Karagiannis I, et al. The burden of extra-pulmonary and meningitis tuberculosis: An investigation of national surveillance data, Germany, 2002 to 2009. Euro Surveill 2013; 18:20436.

2. Merkler AE, Reynolds AS, Gialdini G, et al. Neurological complications after tuberculous meningitis in a multi-state cohort in the United States. J Neurol Sci 2017; 375:460-463.

3. Zignol M, Sigmanidis C, Falzon D, et al. Multidrug-resistant tuberculosis in children: evidence from global surveillance. Eur Respir $\mathrm{J}$ 2013; 42:701-707.

4. Solomons RS, Visser DH, Friedrich SO, et al. Improved diagnosis of childhood tuberculous meningitis using more than one nucleic acid amplification test. Int J Tuberc Lung Dis 2015; 19:74-80.

5. WHO. World Health Organization. Automated real-time nucleic acid amplification technology for rapid and simultaneous detection of tuberculosis and rifampicin resistance: Xpert MTB/RIF assay for the diagnosis of pulmonary and extra pulmonary TB in adults and children:policy update 2013; https://apps.who.int/iris/handle/10665/112472

6 Murthy J.M.K. Tuberculous meningitis: the challenges. Neurol India 2010; 58:716-722.

7. Blakemore R, Story E, Helb D, et al. Evaluation of the analytical performance of the Xpert MTB/RIF assay. J Clin Microbiol 2010; 48:2495-2501.

8. Barnard M, Gey van Pittius NC, van Helden PD, Bosman M, Coetzee G, Warren RM. The diagnostic performance of the GenoTypeMTBDRplus Version 2 line probe assay is equivalent to that of the Xpert MTB/RIF assay. $J$ Clin Microbiol 2012; 50:3712-3716.
9. RNTCP. Revised National Tuberculosis Control Programme. Standard operating procedures for Mycobacteriology laboratory. ICMR 2010; http://nirt.res.in/pdf/bact.SOP.pdf.

10. Xpert MTB/RIF kit inserts. http://www.cepheid.com/manageddownloads/xpertmtb-rif-english-package-insert-301-1404-rev-bfebruary-2015.pdf.

11. Boehme CC, Nabeta $P$, Hillemann $D$, et al. Rapid molecular detection of tuberculosis and rifampin resistance. N Engl J Med 2010; 363:10051015.

12. Siddiqi SH, Rüsch-Gerdes S. MGIT procedure manual. Foundation for Innovative New Diagnostics, Geneva, Switzerland 2006 Jul:41-51.

13. Martin A, Bombeeck D, Mulders W, Fissette K, De Rijk P, Palomino JC. Evaluation of the TB Ag MPT64 Rapid test for the identification of Mycobacterium tuberculosis complex. Int J Tuberc Lung Dis 2011; 15:703-705.

14. Kumar VG, Urs TA, Ranganath RR MPT 64 Antigen detection for Rapid confirmation of $M$. tuberculosis isolates. BMC Research Notes 2011; $4: 1-4$

15. GenoTypeMTBDRplus, VER 2.0, Instructions for Use, IFU-304A-02, Hain Lifesciences $\mathrm{GmbH}$, Germany 2012.

16. Gardee $\mathrm{Y}$, Dreyer AW, Koornhof HJ, et al. Evaluation of the Genotype ${ }^{\circledR}$ MTBDRs| ${ }^{\circledR}$ VER 2.0 assay for second-line drug resistance detection of Mycobacterium tuberculosis isolates in South Africa. J Clin Microbiol 2017; 55:791-800.

17. Raizada N, Sachdeva KS, Swaminathan S, et al. Piloting Upfront Xpert MTB/RIF Testing on Various Specimens under Programmatic Conditions for Diagnosis of TB \& DR-TB in Paediatric Population. PLoS ONE 2015; 10:e0140375.

18. Bhatia R, Dayal R, Jindal S, Agarwal D, Goyal A. GeneXpert for Diagnosis of Tubercular Meningitis. Indian J Pediatr 2016; 83:1353-1355.

19. Bala S, Goyal S. Study on cartridge based nucleic acid amplification test in children with Neurotuberculosis at RNT Medical College, Udaipur, Rajasthan, India. Int J Contemp Pediatr 2019; 6:1588.

20. Krishnakumariamma K, Ellappan K, Muthuraj M, Tamilarasu K, Kumar SV, Joseph NM. Molecular diagnosis, genetic diversity and drug sensitivity patterns of Mycobacterium tuberculosis strains isolated from tuberculous meningitis patients at a tertiary care hospital in South India. PloS ONE 2020; 15:e0240257. 
21. Kohli M, Schiller I, Dendukuri N, et al. Xpert MTB/RIF assay for extra-pulmonary tuberculosis and rifampicin resistance. Cochrane Database Syst Rev 2018; 8:CD012768.

22. Banada PP, Sivasubramani SK, Blakemore R, et al. Containment of bioaerosol infection risk by the Xpert MTB/RIF assay and its applicability to pointof-care settings. J Clin Microbiol 2010; 48:35513557.

23. Nhu NT, Heemskerk D, Chau TT, et al. Evaluation of GeneXpert MTB/RIF for diagnosis of tuberculous meningitis. J Clin Microbiol 2014; 52:226-233.

24. Das PK, Ganguly SB, Mandal B. Early diagnosis of smear-negative childhood pulmonary tuberculosis and its substantial yield in gastric lavage/aspirates through cartridge-based nucleic acid amplification test (Xpert Mycobacterium tuberculosis/rifampicin assay). Biomed Biotechnol Res J 2019; 3:258-263.

25. Padayatchi N, Bamber S, Dawood H, Bobat R. Multidrug-resistant tuberculous meningitis in children in Durban, South Africa. Pediatr Infect Dis J 2006; 25:147-150.

26. Santiago-García B, Blázquez-Gamero D, Baquero-Artigao $\mathrm{F}$, et al. Pediatric extrapulmonary tuberculosis. Pediatr Infect Dis J 2016; 35:11751181.

27. Shah I, Chilkar S. Clinical profile of drug resistant tuberculosis in children. Indian Pediatr 2012; 49:741-744.

28. Al-Mutairi NM, Ahmad S, Mokaddas E, Eldeen HS, Joseph. Occurrence of disputed rpoB mutations among Mycobacterium tuberculosis isolates phenotypically susceptible to rifampicin in a country with a low incidence of multidrug-resistant tuberculosis. BMC Infect Dis 2019; 19:3. 